BULL. AUSTRAL. MATH. SOC.

\title{
Maximal sum-free sets in finite abelian groups, $V$
}

\section{H.P. Yap}

Let $\lambda(G)$ be the cardinality of a maximal sum-free set in a group $G$. Diananda and Yap conjectured that if $G$ is abelian and if every prime divisor of $|G|$ is congruent to 1 modulo 3 , then $\lambda(G)=|G|(n-1) / 3 n$ where $n$ is the exponent of $G$. This conjecture has been proved to be true for elementary abelian p-groups by Rhemtul la and street and for groups $G=Z_{p} \oplus Z_{p}$ by Yap. We now prove this conjecture for groups $G=Z_{p q} \oplus Z_{p}$ where $p$ and $q$ are distinct primes.

Let $G$ be an additive group with non-empty subsets $S$ and $T$. Let $S \pm T=\{s \pm t ; s \in S, t \in T\}$ and let $|S|$ be the cardinality of $S$. We say that $S$ is sum-free in $G$ if $(S+S) \cap S=\varnothing$ and that $S$ is a maximal sum-free set in $G$ if $|S| \geq|T|$ for every $T$ sum-free in $G$. We denote by $\lambda(G)$ the cardinality of a maximal sum-free set in $G$.

Exact values $\lambda(G)$ for all finite abelian groups $G$, except when every prime divisor of $|G|$ is congruent to 1 modulo 3 , were determined by Diananda and Yap [1]. In this exceptional case,

$$
|G|(n-1) / 3 n \leq \lambda(G) \leq(|G|-1) / 3
$$

where $n$ is the exponent of $G$. It is conjectured that in this exceptional case, $\lambda(G)$ equals its lower bound [1]. Rhemtulla and Street [3] prove this conjecture for elementary abelian p-groups. Yap [4] proves this conjecture for groups $G=z_{p}{ }^{2} \oplus z_{p}$.

Received 1 August 1975. 
We now prove this conjecture for groups $G=z_{p q} \oplus Z_{p}$ where $p$ and $q$ are distinct primes. We shall in fact prove the following

THEOREM 1. Let $G=z_{p q} \oplus Z_{p}$ where $p=3 k+1$ and $q=32+1$ are distinct primes. Then $\lambda(G)=p(k q+l)$.

Proof. We shall adopt the definitions and notations given in [4] and [5]. In particular, we let

$$
\begin{gathered}
H_{0}=[(p, 0)] \oplus[(0,1)], H_{i}=[(1, i)], i=1,2, \ldots, p, \\
L=[(q, 0)] \oplus[(0,1)], \text { and } K=[(p, 0)] .
\end{gathered}
$$

From the above definitions, we observe that each $H_{i}$ is a cyclic group of order $p q, H_{i} \cap H_{j}=K$ for each $i \neq j, L$ is an elementary p-group such that $L_{i}=L \cap H_{i}$ is a group of order $p$ for every $i=0,1, \ldots, p$, and $G=\bigcup_{i=0}^{p} H_{i}$.

From (1) we have

$$
p(k q+l)=p(l p+k) \leq \lambda(G) \leq\left(q p^{2}-1\right) / 3 .
$$

Now let $S$ be a maximal sum-free set in $G$. Let $\left|S \cap H_{i}\right|=2 p+\lambda_{i}=k q+\mu_{i}, i=0,1, \ldots, p$, $\lambda=\max \left\{\lambda_{i} ; i=0,1, \ldots, p\right\}, \mu=\max \left\{\mu_{i} ; i=0,1, \ldots, p\right\}$, and let $H \in\left\{H_{0}, H_{1}, \ldots, H_{p}\right\}$ be such that

$$
|S \cap H|=z_{p}+\lambda=k q+\mu, \lambda \leq k, \quad \mu \leq \imath .
$$

(The fact that $\lambda \leq k$ and $\mu \leq 2$ follows from (1).)

By definition we have, for each $i=0,1, \ldots, p$,

$$
\lambda_{i}=k-\imath+\mu_{i} \text { and, in particular, } \lambda=k-\imath+\mu .
$$

From (1) we have

$$
|S \cap K|=m, \quad 0 \leq m \leq l \text {. }
$$

By looking at the distribution of the elements of $S$ in the subgroups $H_{i}$, we have 


$$
\begin{aligned}
& \text { Maximal sum-free sets } \\
& \begin{aligned}
|S| & =\sum_{i=0}^{p}\left|S \cap H_{i}\right|-p|S \cap K| \\
& =(p+1) l_{p}+\sum_{i=0}^{p} \lambda_{i}-p m .
\end{aligned}
\end{aligned}
$$

If $|S|>p(2 p+k)$, then

$$
\sum_{i=0}^{p} \lambda_{i}>p(k-\tau+m) .
$$

We also have

$$
\begin{aligned}
|S| & \leq(p+1)(k q+\mu)-p m \\
& =k p q+p(\mu-m)+2 p+k-z+\mu .
\end{aligned}
$$

Thus if $\mu<m$, then (7) contradicts (2). Hence

$$
\mu \geq m \text {. }
$$

Next, let $K_{0}=K, \quad H=\bigcup_{i=0}^{p-1} K_{i}$ where $K_{i}=x_{i}+K$ are distinct cosets of $K$ in $H$ such that $x_{1}+x_{1}=x_{2}$, $x_{1}+x_{2}=x_{3}, \ldots, x_{i} \in H \cap L$. Let $x_{i}+S_{i}=S \cap K_{i}$. Then we have

$$
m(H)=\max \left\{\left|S_{i}\right| ; i=1,2, \ldots, p-1\right\} \geq 2+1,
$$

for otherwise $k q+\mu=|S \cap H| \leq(p-1) z+m=k q-k+m$ which contradicts (8).

If $m(H) \geq 2+2$, then we can show that at least one of $S_{i}$ is empty (see the proof of Theorem 4 [5]) and following the proof of Theorem 2 [4], we can show that $\lambda(G)$ equals its lower bound given in (2). Thus if (10)

$$
|S|>p(k q+Z) \text {, then } m(H)=Z+1 \text {. }
$$

Suppose $\mu>m$. Then

$$
k q+\mu-m \geq k q+1=(k+1)(2+1)+(2 k-1) z,
$$

and applying the theorem of Cauchy-Davenport (see [2]) to $S_{i}$, we get a contradiction (for details of the proof of this part, see the proof of Theorem 4 [5]). Hence if

$$
|S|>p(k q+l) \text {, then } \mu=m \text {. }
$$


Substituting (11) into (6) we get

$$
\sum_{i=0}^{p} \lambda_{i}>p \lambda
$$

from which it follows that if

$$
|S|>p(k q+i) \text {, then } \lambda_{i}>0 \text { for each } i=0,1, \ldots, p \text {. }
$$

Next, from (11), we have

$$
k q+\mu=|S \cap H|=k(z+1)+2 k z+m,
$$

from which it follows that there are $k$ elements in $\left\{s_{1}, s_{2}, \ldots, s_{p-1}\right\}$ each of which has cardinality $l+1$. Suppose $\left|S_{i}\right|=l+1$. Then applying the theorem of Cauchy-Davenport to $S_{i}+S_{i}$ and noting that $\left(S_{i}+S_{i}\right) \cap S_{2 i}=\emptyset$, we know that $S_{2 i}=K \backslash\left(S_{i}+S_{i}\right)$, the set complement of $S_{i}+S_{i}$ with respect to $K$. Thus, we have either $0 \in S_{i}$ or $0 \in S_{2 i}$; that is, either $x_{i} \in S$ or $x_{2 i} \in S$. Hence $|S \cap L \cap H|=k$.

Let $I=\left\{i_{1}, i_{2}, \ldots, i_{p+1}\right\}$ be such that for each $i \in I, \lambda_{i}<\lambda$, and for each $i \in\{0,1, \ldots, p\} \backslash I, \lambda_{i}=\lambda$. Let $H_{j}=\bigcup_{i=0}^{p-1} K_{i j}$ where $K_{i j}=x_{i j}+K$ are distinct cosets of $K$ in $H_{j}$ such that $x_{1 j}+x_{1 j}=x_{2 j}, x_{1 j}+x_{2 j}=x_{3 j}, \ldots, x_{i j} \in L_{j}$. Let $x_{i j}+S_{i j}=S \cap K_{i j}$. Then for each $j \in I$, we have

$$
\begin{aligned}
k q+\mu_{j} & =\left|S \cap H_{j}\right| \leq r_{j}(z+1)+\left(p-r_{j}-1\right) \downarrow+m \\
& =k q-k+r_{j}+m,
\end{aligned}
$$

from which it follows that $r_{j} \geq k+\mu_{j}-m$.

By the same reasoning as in showing that $|S \cap L \cap H|=k$, we can show that if $\left|S_{i j}\right|=z+1$, and if $\left|S_{(2 i) j}\right| \leq z-1$, then there are at least $r_{j}+1$ elements in $\left\{S_{1 j}, \ldots, S_{(p-1) j}\right\}$ each of which has cardinality $\ell+1$. Thus, by the replacement process, we have $\left|s \cap L_{j}\right| \geq r_{j}$. 
Putting the above results together, we have

$$
\begin{aligned}
|S \cap L| & \geq(p-r) k+(r+1)(\dot{k}-m)+\sum_{j \in I} \mu_{j} \\
& =(p-r) k+(r+1)(k-m)+(r+1)(z-k)+\sum_{j \in I} \lambda_{j} \\
& =k p+(r+1)(z-m-k)+k+r \lambda \\
& =k p+(r+1)(z-m-k+\lambda)+k-\lambda \\
& =k p+k-\lambda .
\end{aligned}
$$

Finally, we decompose $H_{i}$ as a union of $q \operatorname{cosets} L_{i j}$, $j=1, \ldots, q$ of $L_{i}$. If for some $i$,

$$
\max \left\{\left|S \cap L_{i j}\right| ; j=1,2, \ldots, q\right\} \geq k+2 \text {, }
$$

then following the proof of Theorem $2[4]$, we can show that $\lambda(G)$ equals the lower bound given in (2). Otherwise, from

$$
2 p+\lambda_{i}=\left|S \cap H_{i}\right|=2(k+1)+22 k+\lambda_{i},
$$

it follows that $\left|S \cap L_{i}\right| \geq \lambda_{i}$ and thus $|S \cap L| \geq \sum_{i=0}^{p} \lambda_{i}>p \lambda$ which cannot be true if $\lambda=k$ (see Theorem 1 [3]). Consequently, if

$$
|s|>p(k q+z) \text {, then } \lambda<k \text {. }
$$

From (14) and (15), we have

$$
|S \cap L|>k p \text {, }
$$

which again contradicts Theorem 1 [3].

Hence $\lambda(G)=p(k q+l)$.

\section{References}

[1] Palahenedi Hewage Diananda and Hian Poh Yap, "Maximal sum-free sets of elements of finite groups", Proc. Japan Acad. 45 (1969), 1-5.

[2] Henry B. Mann, Addition theorems: The addition theorems of group theory and number theory (Interscience Tracts in Pure and Applied Mathematics, 18. John Wiley, New York, London, Sydney, 1965). 
[3] A.H. Rhemtulla and Anne Penfold Street, "Maximal sum-free sets in finite abelian groups", Bull. Austral. Math. Soc. 2 (1970), 289-297.

[4] H.P. Yap, "Maximal sum-free sets in finite abelian groups IV", Nanta Math. 5 (1972), no. 3, 70-75.

[5] H.P. Yap, "Maximal sum-free sets in finite abelian groups, III", $J$. Number Theory 5 (1973), 293-300.

Department of Mathematics,

University of Singapore,

Singapore. 